\title{
UN DISEÑO DE MOSAICO ROMANO EN LA CERÁMICA SEVILLANA DEL SIGLO XVI
}

\author{
POR M. a JESÚS SANZ
}

Se analizan dos mosaicos sevillanos del siglo XVI y su posible relación con un diseño ya utilizado en el Mundo Romano. Se trata también de ver las distintas influencias que existían en los diseños artísticos de la primera mitad del siglo XVI, y en especial en la cerámica, así como la variedad de técnicas utilizadas.

The possible connection between two Sevillian mosaics and a Roman one from Mérida is studied in this paper. Also are examined the various influences detected in 16th century ceramics in general, as well as in these two pieces. The different techniques used in the mosaics are also studied.

Aunque existen bastantes trabajos sobre cerámica sevillana, no puede decirse que el conjunto de la producción hispalense se halle estudiada durante su largo período de desarrollo. Por el contrario hay abundantes y completos estudios sobre la cerámica valenciana, la catalana, o la de Talavera, escuelas todas ellas de gran importancia, pero en absoluto superiores a la sevillana, malagueña o granadina, que no disponen de estudios tan completos como las anteriormente mencionadas.

Sobre la cerámica sevillana se han publicado monografías puntuales de algunos artistas como Pisano ', algunos artículos sobre cerámica del siglo XVII y del $\mathrm{XVIII}^{2}$, catálogos de exposiciones ${ }^{3}$, y finalmente trabajos sobre los siglos XIX y

1. MORALES, A.: Francisco Niculoso Pisano, Sevilla, 1977.

2. PlegueZUElo, A.: Azulejo Sevillano: Catálogo del Museo de Artes y Costumbres Populares de Sevilla, Sevilla, 1989.

3. PLEGUEZUELO, A.: "Sevilla y Talavera entre la colaborción y la competencia", Laboratorio de Arte, Homenaje al Profesor Bemales, tomo I, Sevilla, 1992, págs. 275-294. 
$\mathrm{XX}$, que aún no se hallan publicados. Naturalmente para todos ellos han sido fundamentales los estudios previos de Gestoso ${ }^{4}$ y de Sancho Corbacho ${ }^{5}$, pero a pesar de todo no existe hoy aún una obra de conjunto, o al menos de una amplia etapa determinada.

La utilización de la cerámica como revestimiento arquitectónico en Andalucía es una tradición que, partiendo de la época musulmana, llega hasta nuestros días, y por ello el campo de trabajo resulta inmenso a la hora de hacer un estudio sobre él.

La importancia del revestimiento cerámico en la arquitectura sevillana a lo largo del Medievo cristiano, y de la primera Edad Moderna quizá no sea bastante patente al estudioso del arte sevillano, porque casi todos los edificios han sido bastante trasformados a partir del siglo XVIII. En esta última época se cambiaron muchas solerías -entre ellas la de la catedral-, sustituyendo los suelos de olambrilla por los de mármol; incluso en nuestros días se ha seguido realizando esta práctica, pues la solería primitiva mudéjar de la capillita de Santa María de Jesús fue cambiada por una de mármol en 1963. Otro motivo por el que estos revestimientos cerámicos han desaparecido ha sido la fragilidad del mismo material cerámico, que con el tiempo y el uso continuado se desgastan, rompen y ondulan, por lo que se hace necesario sustituirlos, y al hacerlo se utilizan materiales más resistentes.

El tema que aquí nos ocupa es muy concreto y se halla relacionado, más que con la técnica cerámica, con los motivos que inspiraban las composiciones de los grandes paneles, que revestían los principales edificios sevillanos a lo largo del siglo XVI. Sabemos que en primer cuarto del siglo el predominio del azulejo de tipo mudéjar era absoluto, tanto en las técnicas empleadas -cuenca y cuerda seca-, como en los motivos decorativos, recordemos los maravillosos lienzos de la Casa de Pilatos, varios zócalos del refectorio y el patio de la Cartuja de Las Cuevas, etc. En estas fechas sólo la figura de Niculoso Pisano destaca como innovador de las técnicas, introduciendo la cerámica pisana o pintada.

En el segundo tercio del siglo tenemos bastantes noticias documentales de ceramistas, aportadas fundamentalmente por Gestoso, cuyas obras se conservan en parte, pero sobre todo es el el último tercio del siglo en el que aparece más documentación de azulejeros, y en el que hay más obras conservadas. Desde el segundo tercio del siglo se va a implantar definitivamente la cerámica pisana, que poco a poco irá desplazando a las antiguas técnicas, aunque éstas nunca desaparecerán del todo. Es este período uno de los más creativos del Renacimiento Español en todas las artes, y también en la cerámica, donde puede decirse

4. GESTOSO, J.: Barros vidriados sevillanos, Sevilla, 1904.

5. SANCHO CORBACHO, A.: Cerámica sevillana del siglo XVI, de cuenca, Sevilla, 1953. 
que se emplean casi todas las técnicas conocidas en el momento. Se empleará la cuenca $o$ arista en gran abundancia, más restringidamente se empleará la cuerda seca, irrumpirá con gran fuerza la cerámica pisana, y finalmente se empleará también el mosaico cerámico, a la manera musulmana.

Los paneles cerámicos que nos ocupan son muy semejantes, teniendo las mismas líneas técnicas, y los mismos motivos ornamentales en su espacio principal, siendo estos poco habituales en la cerámica de su tiempo.

Se trata del rosetón que adorna la entrada de los pies de la iglesia del monasterio de La Cartuja de Las Cuevas (fig.1), y el del suelo del Pabellón de Carlos $V$ en el Alcázar de Sevilla (fig.2). En ambas superficies se coloca un gran anillo alrededor de un núcleo central, en el caso del testero de La Cartuja, alrededor de la gran ventana circular, y en el caso del pabellón, alrededor de una fuente de mármol. Este gran anillo está formado por un mosaico de piezas rómbicas esmaltadas, que se colocan en varios círculos concéntricos alrededor del núcleo central -ventana o fuente-, y van aumentando de tamaño a medida que se alejan del centro. En el caso de La Cartuja los colores utilizados son blanco, negro, azul-celeste y melado, y en el caso del Alcázar se añade además el verde, que como puede verse son los colores tradicionales del azulejo mudéjar. En el rosetón de La Cartuja los colores se hallan colocados en líneas radiales, alternando con el blanco los otros tres colores, y terminando en triángulos blancos tanto en la circunferencia exterior como en la interior. En el Alcázar por el contrario los colores se distribuyen en círculos concéntricos, partiendo del melado, en la circunferencia menor, y pasando por el negro, verde y azul (fig.3). Estas líneas concéntricas de color se alternan con otras de rombos blancos, color que, como en el caso anterior, ocupa el inicio y final con una línea de triángulos. En La Cartuja las líneas concéntricas de rombos son cinco -dos blancas y tres de color-, más las dos de triángulos de los extremos. En el Alcázar, aún siendo una superficie menor, son siete -cuatro de color y tres blancas-, más los dos círculos de triángulos.

Este tema ornamental de rombos de colores formando círculos concéntricos es muy poco habitual en la cerámica del XVI, pero sin embargo hemos hallado un claro antecedente en un mosaico romano de Mérida, que está documentado como obra de los siglos III o $\mathrm{IV}^{6}$, se halla en la llamada Casa del Anfiteatro (fig.4), formando parte de un ancho vestíbulo o atrio en el que figuran tres alfombras cuadradas con un círculo interior, siendo uno de ellos el de los rombos. Los rombos aquí están formados por teselas cúbicas, como es habitual en el mosaico romano llamado "opus tesellatum", pero la impresión que da al espectador es de ser un "opus sectile", es decir, formado por placas de color recortadas.

6. BLANCO FREJEIRO, A.: Mosaicos romanos de Mérida, Madrid, 1978, págs. 18-19 y 42. 
El material empleado es caliza blanca, roja y gris, alternándose el blanco con las otras líneas de color, que se organizan en círculos concéntricos, como en el Alcázar, pero que aquí son trece -siete blancas y seis de color-, más los dos círculos exterior e interior de triángulos, gris y rojo respectivamente. Estas líneas de rombos enmarcan en el centro una estrella de seis puntas rodeada de una cenefa en forma de cable, que se repite en el borde exterior. Los triángulos formados por la conjunción del cuadrado circunscrito al círculo se ocupan con diseños de ánforas cubiertas y roleos.

En los mosaicos cerámicos sevillanos también existen partes exteriores e interiores decoradas, pero éstas no guardan relación alguna con el mosaico de Mérida, sino que se corresponden con los temas de su época. El de La Cartuja contiene tres círculos -uno interior y dos exteriores-, de diseño vegetal muy menudo, asi como una crestería como remate, en la línea más exterior, ésta de tipo manierista. Este rosetón de La Cartuja tiene además un seudogablete en la parte superior que contiene todas las líneas concéntricas decorativas del óculo, excepto la del mosaico en cuestión, rellenando su triángulo interior con penachos vegetales como los de la crestería externa.

El mosaico del suelo del Pabellón de Carlos V, llamado tradicionalmente "Cenador de La Alcoba", resulta de un diseño, y de unas técnicas mucho más complicadas.

Sobre este edificio y su construcción nos dio las primeras noticias Gestoso ${ }^{7}$, y más tarde se realizaron diversos trabajos sobre é $1^{8}$ que han aclarado bastantes puntos de este interesante edificio. Se trata de un templete cuadrado con cubierta de cúpula de madera acasetonada, que contiene un puerta en uno de los lados y ventanas en los otros tres. Alrededor corre una galería o porche sostenida por columnas, entre cuyos fustes se ubica un murete con banco. Tanto este murete como las paredes exteriores e interiores del edificio van cubiertas de azulejos, y así mismo en el suelo del espacio interior encontramos solería de mosaico y olambrilla, que es la pieza que a nosotros nos interesa.

Según Jiménez el edificio pudo ser una cubba abbadí dedicada a la oración o al enterramiento, que más tarde quedó dentro de la muralla almohade. En la época de Carlos $\mathrm{V}$, se renovó junto con el jardín exterior, probablemente para que sirviera de fresco descanso en el caluroso verano sevillano. El autor de la

7. GESTOSO, J.: Sevilla monumental y artística, tomo I, Sevilla, 1989, págs. 400, 513, 514 y 516.

8. JIMÉNEZ, A.: "Dibujos de arquitectura sevillana 1. El mirador de la Alcoba", en Revista de Arte Sevillano, n.․ 2, Sevilla, 1982, págs. 51-56. Este trabajo presenta unos magníficos dibujos de planta y alzado, así como de los capiteles y diseño del suelo. Posteriormente se ha publicado otro trabajo en el que se recogen datos anteriores y se añaden algunos nuevos sobre la construcción del edificio. Véase MARÍN FIDALGO, A.: El Alcázar de Sevilla bajo los Austrias, Sevilla, 1987, págs. 172-176. 
completa renovación parece ser que fue el maestro de obras Juan Hernández o Fernández, cuyo nombre y fecha de terminación -1546- figura en el suelo del edificio. Según el mencionado investigador este Juan Hernández era maestro mayor de obras del Alcázar desde 1543, habiendo heredado el cargo de su padre del mismo nombre, aunque parece ser que éste era un moro convertido cuyo anterior nombre había sido Hamete el Cobexí ${ }^{9}$

Salvo las columnas de mármol del corredor exterior, de origen italiano, el resto de la construcción está dentro de la más pura tradición mudéjar en lo que a materiales y técnicas se refiere, aunque naturalmente la influencia del Renacimiento italiano se haga sentir en algunos elementos decorativos. El edificio está concebido como una unidad donde suelo, techo y paredes armonizan, relacionándose sus temas ornamentales, aunque los materiales de construcción sean distintos. Parece evidente que el conjunto fue diseñado por una sola persona, que bien pudo ser el mismo Juan Hernández que firma el edificio, especialmente si se atiende al mudejarismo de este, mostrado en el abundante uso del azulejo, en la cubierta de madera, y, en fin, en el magnífico conocimiento de las técnicas requeridas.

El suelo (fig.5) se compone de cuatro partes bien definidas: la fuente de mármol central, el anillo de mosaico que la rodea, los ángulos que quedan entre el cuadrado circunscrito al anillo, y el cuadrado exterior que coincide con la planta del edificio. En cada una de estas partes se han utilizado técnicas y materiales diferentes, componiendo sin embargo todas ellas una pieza absolutamente armónica digna de una mente renacentista, pero que sin embargo participa del rico sentido del color mudéjar.

La parte central presenta una fuente de mármol blanco con un canalillo de desagüe que va hacia la puerta, y rodeándola se halla el mosaico de piezas rómbicas que tratamos. El mosaico, que no constituye un círculo completo, sino que se abre en dos bandas rectangulares bordeando al canalillo de mármol, presenta en estas bandas rectangulares unos rombos totalmente regulares, sin deformación, ni alargamiento como en la parte circular (fig.6), motivo que se repite exactamente igual en la cornisa superior del testero de La Cartuja (fig.7).

Circunscrito al círculo de mosaico se halla un cuadrado tangente a la circunferencia exterior, por lo que sólo quedan de él como espacios visibles cuatro triángulos rectángulos de base curvilínea. De estos triángulos los dos más próximos a la puerta se hallan cortados en uno de los ángulos de su base para dar paso al canalillo. Estos triángulos están recubiertos de mosaico como el anillo interior de rombos, utilizándose en ellos la misma técnica de barro vidriado, pero en este caso la complicación decorativa es mucho mayor, porque en lugar de rombos se

9. JIMÉNEZ, A.: Ob. cit., págs. citadas. 
han utilizado placas recortadas con temas animales y vegetales, que se colocan según la antigua técnica romana llamada "intarsia". Los colores utilizados son los mismos del anillo: blanco, verde, negro y celeste, pero los temas oscilan entre la última tradición gótica y el primer renacimiento. En los dos triángulos mutilados de la entrada, bastante deteriorados por cierto, se halla la fecha y el nombre del arquitecto, a la derecha la fecha -1546- (fig.8), y a la izquierda el nombre IVAN HZ- (fig.9). Ambos datos se hallan dentro de una cartela rectangular rodeada de elementos vegetales de distribución simétrica, entre los que destaca la jarra o bulbo que la sostiene, y dos cuernos laterales de los que brotan inflorescencias. Los dos triángulos del fondo presentan interesantes temas zoomórficos de componente fantástico. El de la derecha, contiene en el centro un tallo vegetal de abundantes y gruesas hojas, que se distribuyen en torno a un eje central, y que se atan por un anillo en su base (fig.10). A los lados aparecen dos animales grandes enfrentados a otros dos más pequeños, que ocupan el ángulo agudo del triángulo. Los animales grandes: un simio pasante que se enfrenta a un pequeño animal, irreconocible por el destrozo del mosaico, y al otro lado un unicornio, que se enfrenta a un pequeño bicornio inclinado ante él (fig.11). El triángulo del fondo-izquierda tiene el mismo diseño que el de la derecha, pero se halla en peores condiciones porque se intentó arreglar en determinado momento colocando varios azulejos sencillos, procedentes de otro lugar, haciendo desaparecer el tallo central. Sin embargo, el simio se conserva mejor, e incluso se aprecia algo del animal que se le enfrenta, que parece ser una especie de dragón con cola en forma de pico de ave (fig.12).

Es interesante comprobar que el tema de el unicornio y el del del simio, de origen medieval, se encuentran también en el mosaico que cubre las paredes exteriores del edificio (fig.13), aunque con tratamiento algo distinto. Esto hace pensar que todos los azulejos salieran del mismo taller, aunque la técnica del suelo -mosaico- fuese distinta de las de las paredes - cuenca-, y que la primera fuese mucho más correcta y refinada en su ejecución que la segunda. En el mosaico del suelo, como hemos visto, se mezclan motivos renacentistas -cartelas y tallos-con motivos de recuerdo medieval -animales fantásticos-, aunque estos los vemos todavía en el primer Renacimiento. Recordemos que un unicornio coloca Niculoso Pisano en el retablo del Alcázar. Estos animales tienen evidentemente un sentido simbólico, pues sabemos que el unicornio, a fines de la Edad Media, era símbolo de la pureza, y el simio de la lujuria, por lo que la escena, que presenta a ambos animales a los lados de un tallo central, podría interpretarse como la bondad y la maldad, o el pecado y la virtud, saliendo del árbol del bien y del mal.

El resto de la solería la compone otro cuadrado mayor que coincide con la planta total del edificio, apreciándose unos salientes en la zona de las ventanas, 
donde el muro se estrecha. La solería aquí muestra los conocimientos geométricos del diseñador, que juega con las figuras geométricas deformándolas a lo largo para conseguir pasar de cuadrados menores a otros mayores sin alterar los trazos iniciales (fig.14). Así una serie de figuras trapezoidales forman una red, de formidable efecto óptico, cuyos espacios centrales se ocupan por figuras de polígonos de varios tipos -rombos y estrellas, preferentemente-, formas mixtilíneas, círculos y óvalos, así como una forma de corazón ${ }^{10}$. La técnica aquí empleada es también la del mosaico, pero en su acepción de "intarsia" como en los ángulos descritos, aunque con diferencias. Aquí se ha usado ladrillo sin esmaltar en dos colores de barro, rojo oscuro para la loseta y rojo amarillento para la red y los perfiles de las pequeñas formas centrales. En las uniones de las líneas de la red se advierten pequeños cuadrados esmaltados en blanco y verde (fig.15). Podría pensarse que la red y los perfiles de las pequeñas formas geométricas estuvieron primitivamente esmaltadas, y que por el uso desapareció el vidriado, pero esto no parece probable, ya que en las zonas deterioradas del mosaico central, y de los ángulos con animales, no se aprecia otro color de barro diferente que el de las zonas donde el vidriado ha desaparecido. Así pues se trata de un originalísimo diseño de suelo inspirado claramente en algún modelo renacentista pensado para mármol, que aquí, por la tradición mudejárica, se pasó a barro cocido.

Con respecto a la autoría, se sabe, por los trabajos ya mencionados, que unos hermanos ceramistas de Triana, Juan y Diego Polido, proporcionaron los azulejos para la construcción, así como otro ollero llamado Pedro Herrera, en el verano de 1543, pero ignoramos que clase de azulejos hicieron cada uno de ellos. En cualquier caso no parece que los complicados diseños del suelo, y la emblemática en él representada fuese obra ni del maestro de obras Juan Hernández, ni de los azulejeros, sino más bien de un diseñador general para todo el edificio que tuviese una cultura humanística y un sentido armónico y proporcional de la ornamentación, cuyo nombre hasta ahora se ignora.

La relación de este mosaico con el de La Cartuja se establece sólo en la parte correspondiente al rosetón de rombos, cuyas piezas podrían proceder del mismo taller de los Pulido, o del de Pedro de Herrera, pero de La Cartuja, en los distintos trabajos publicados, no hay referencia alguna a este mosaico, ni en general a otros artífices que trabajaran en el edificio ${ }^{11}$. Sin embargo, en uno de ellos se dice que el hastial se construyó en 1523, en una ampliación que se hizo

10. JIMÉNEZ, A.: Ob. cit., pág. 56. Se presentan aquí dibujos de todas las piezas.

11. MORALES, A. SANZ, M.J., SERRERA, J.M. y VALDIVIESO, E.: Guía artistica de Sevilla y su provincia, Sevilla, 1981, reedición de 1989, pág. 48 y V.V.A.A.: Historia de la Cartuja de Sevilla. De ribera del Guadalquivir a recinto de la Exposición Universal, Sevilla, 1989. 
de la nave central, y además se reconstruyó la capilla de Santa Ana que estaba recubierta de azulejos, y que se halla a los pies de la iglesia, justo en la parte ampliada ${ }^{12}$, por lo que probablemente en esos años, o poco después, debió colocarse el rosetón del hastial, tan semejante al que se puso en el Alcázar por los años de 1543.

12. CUARTERO Y HUERTA, B.: Historia de La Cartuja de las Cuevas, de Sevilla, y de su filial de Cazalla de la Sierra, Madrid, 1950, 2 tomos, págs. 327-328. 


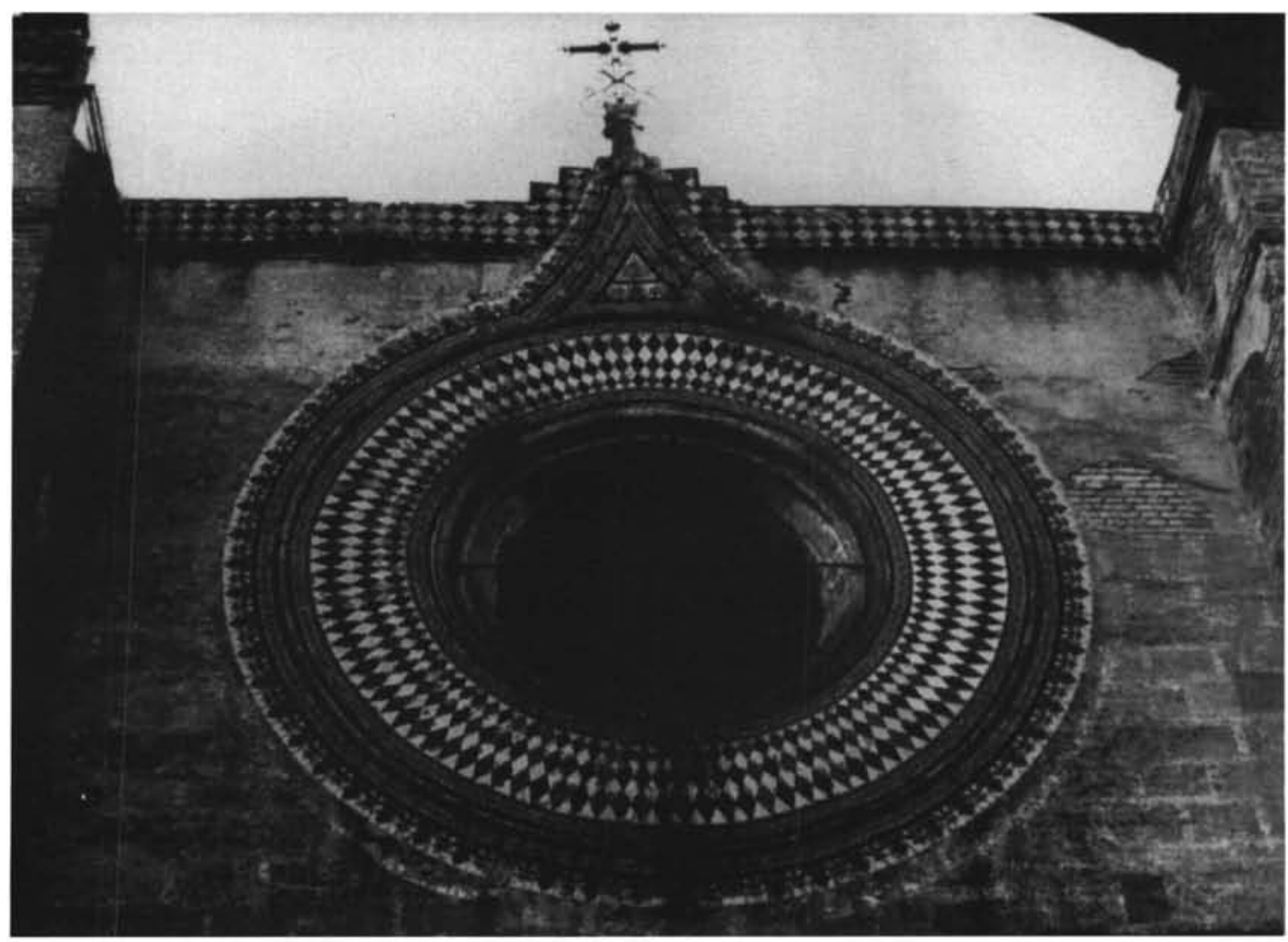




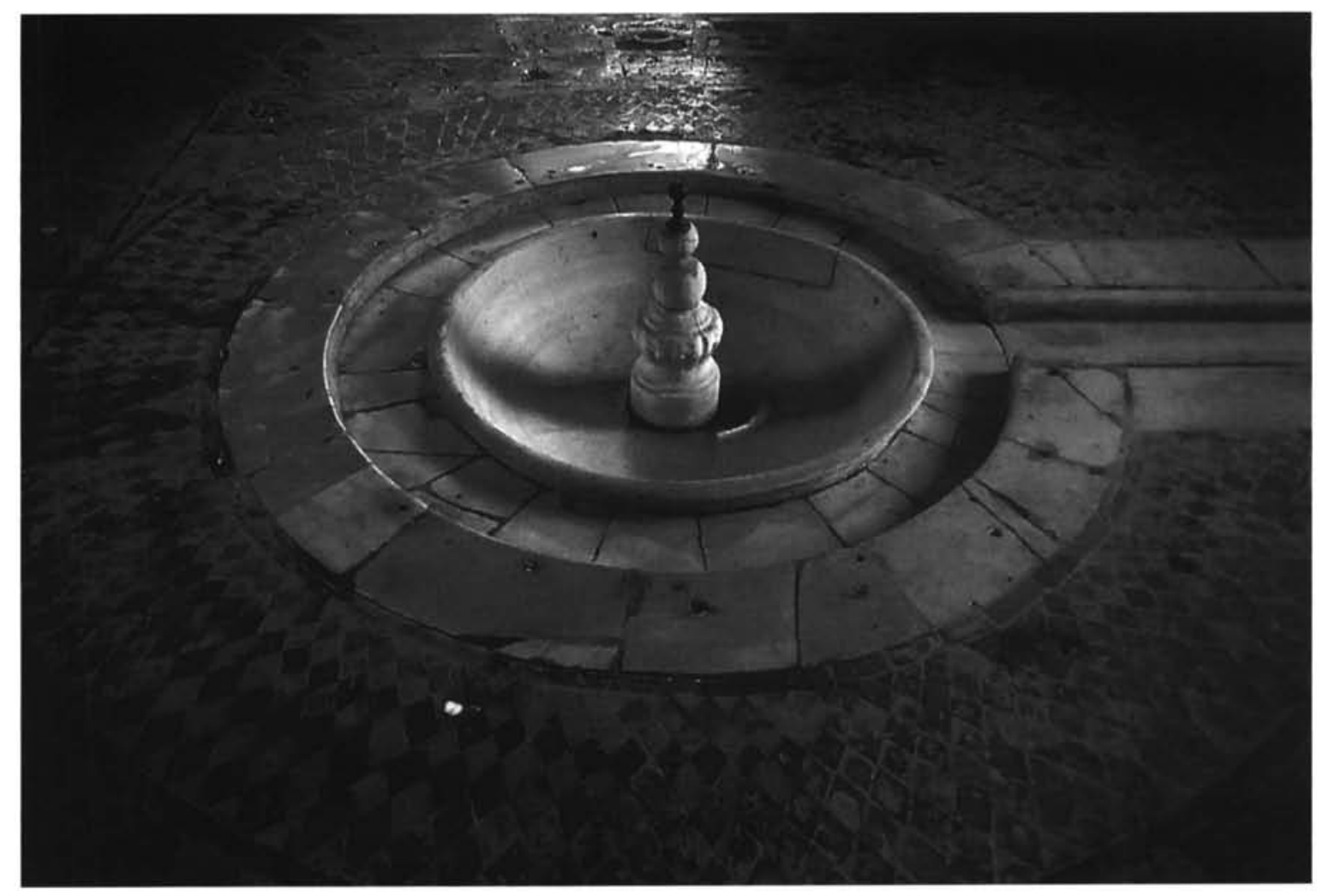



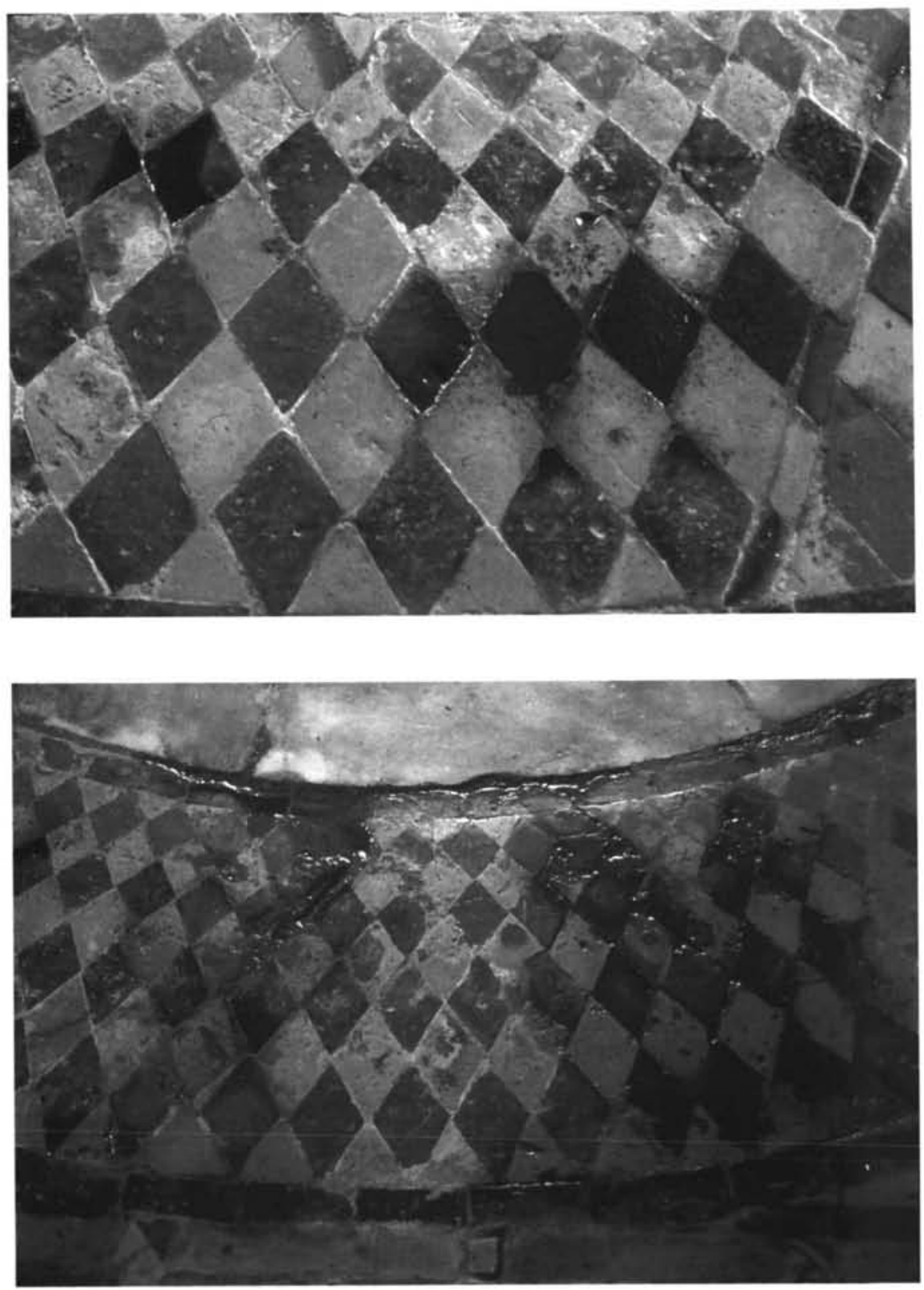

3. Detalles de la figura anterior. 


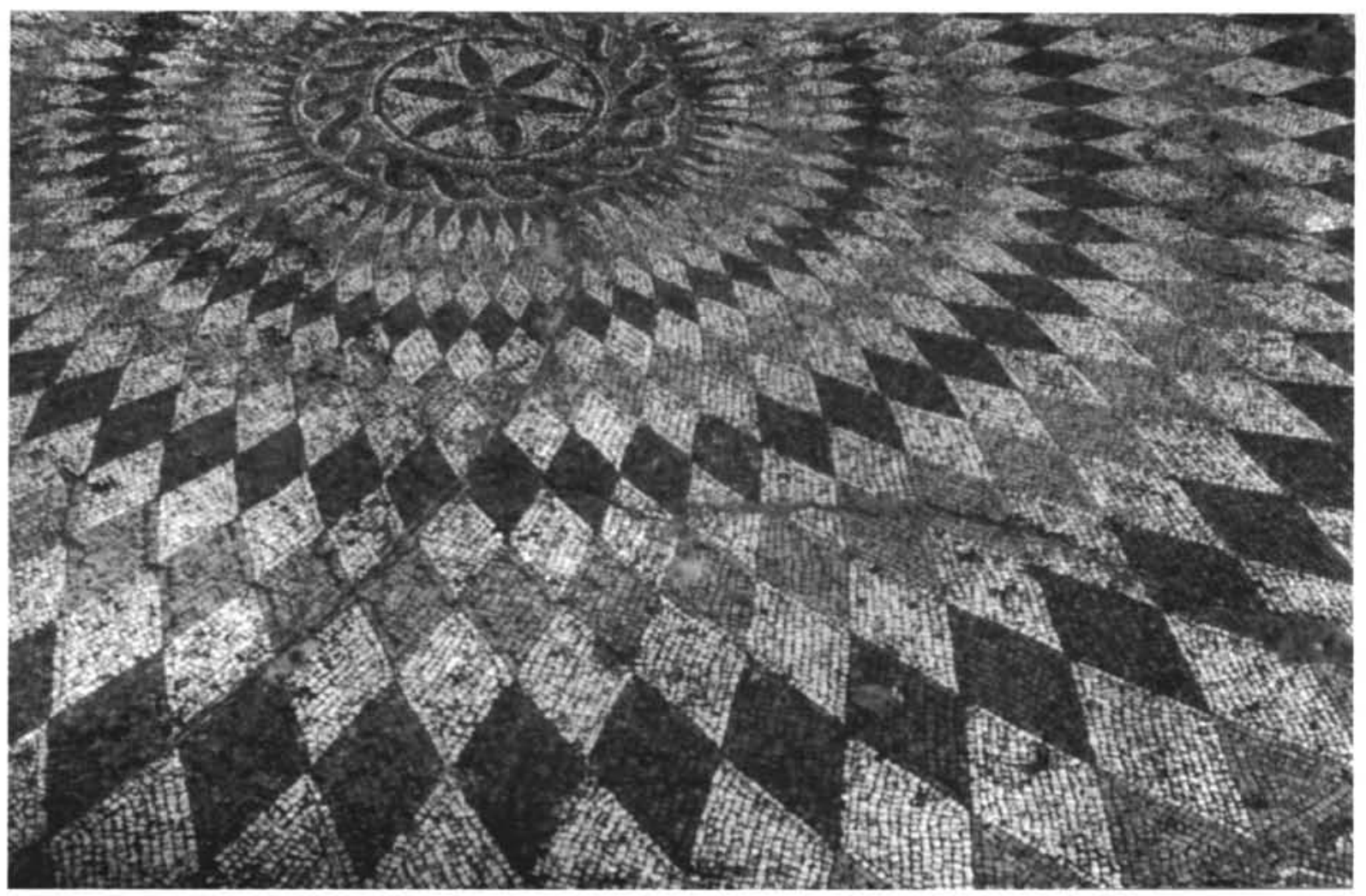

4. Mosaico existente en la llamada Casa del Anfiteatro, en la ciudad romana de Mérida. Siglos III-IV. 


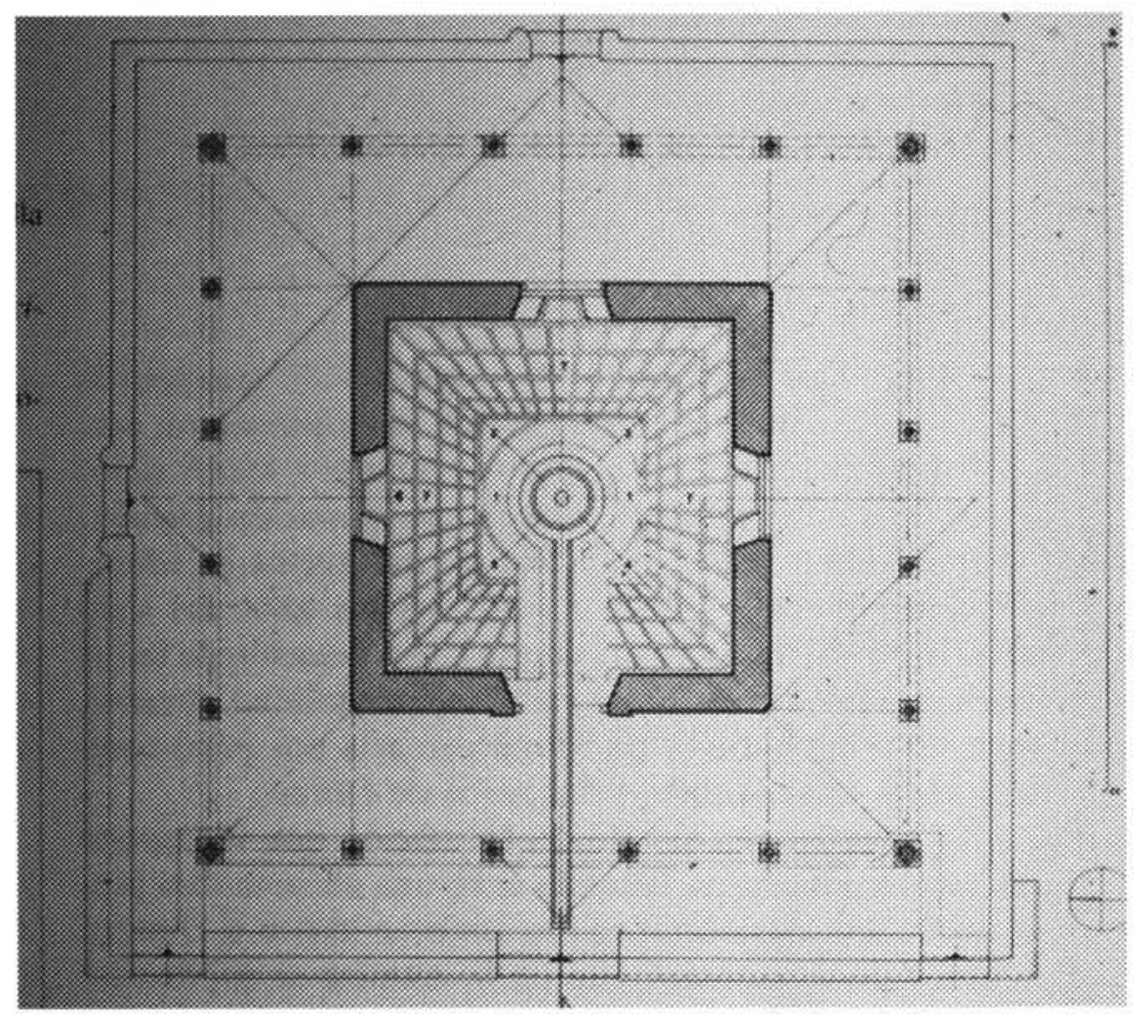

5. Dibujo de la planta del Pabellón de Carlos V. Autor Alfonso Jiménez. 


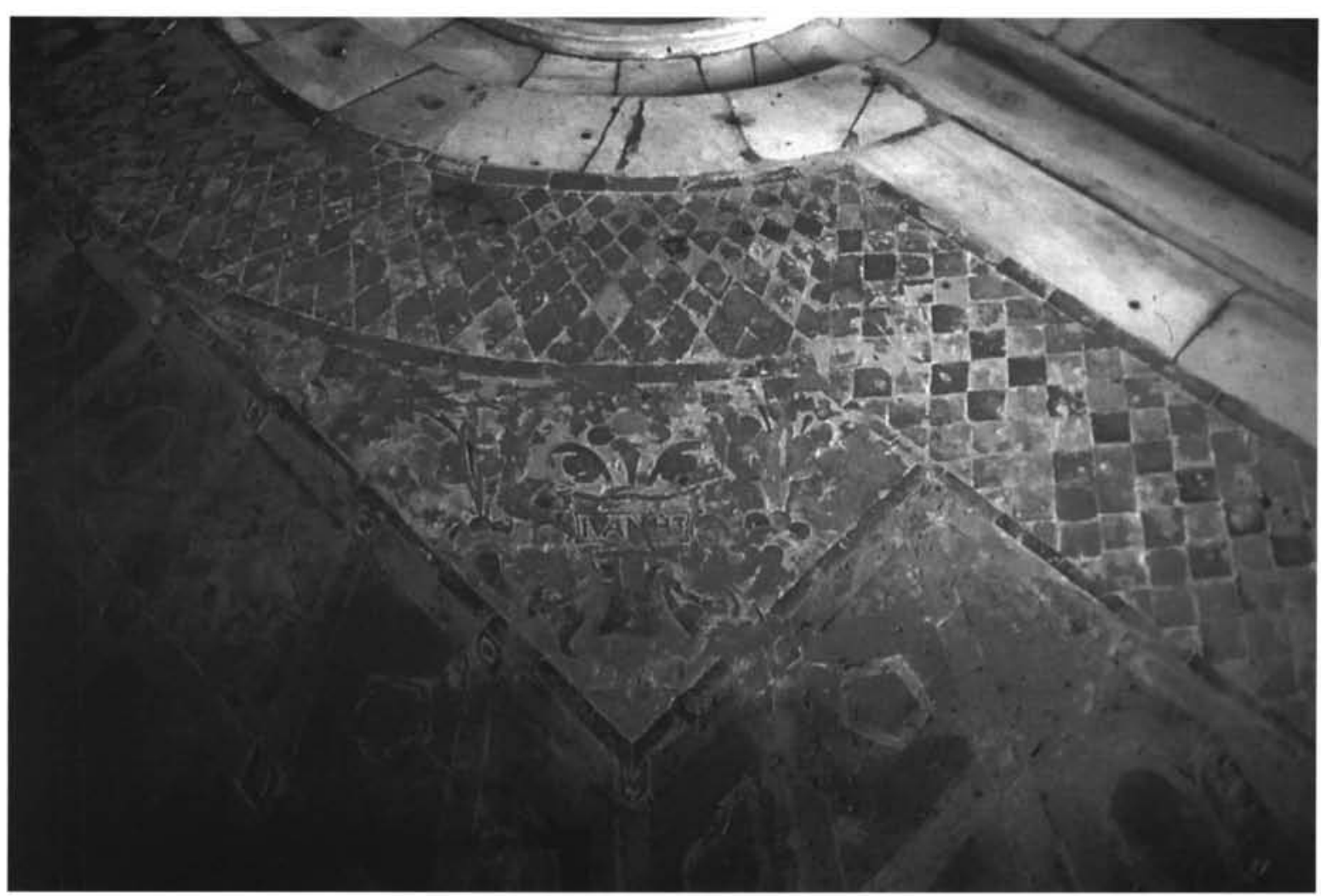




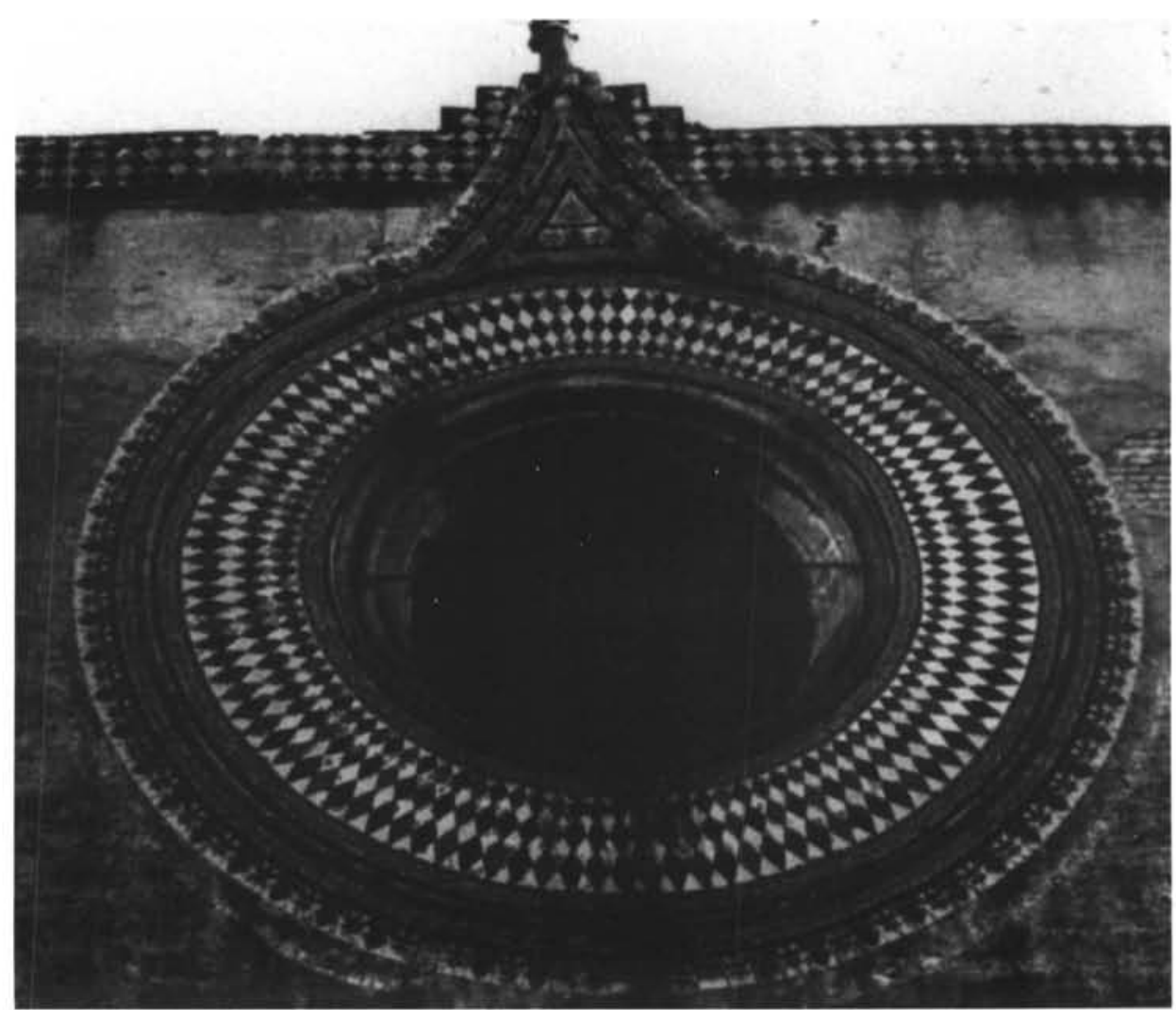




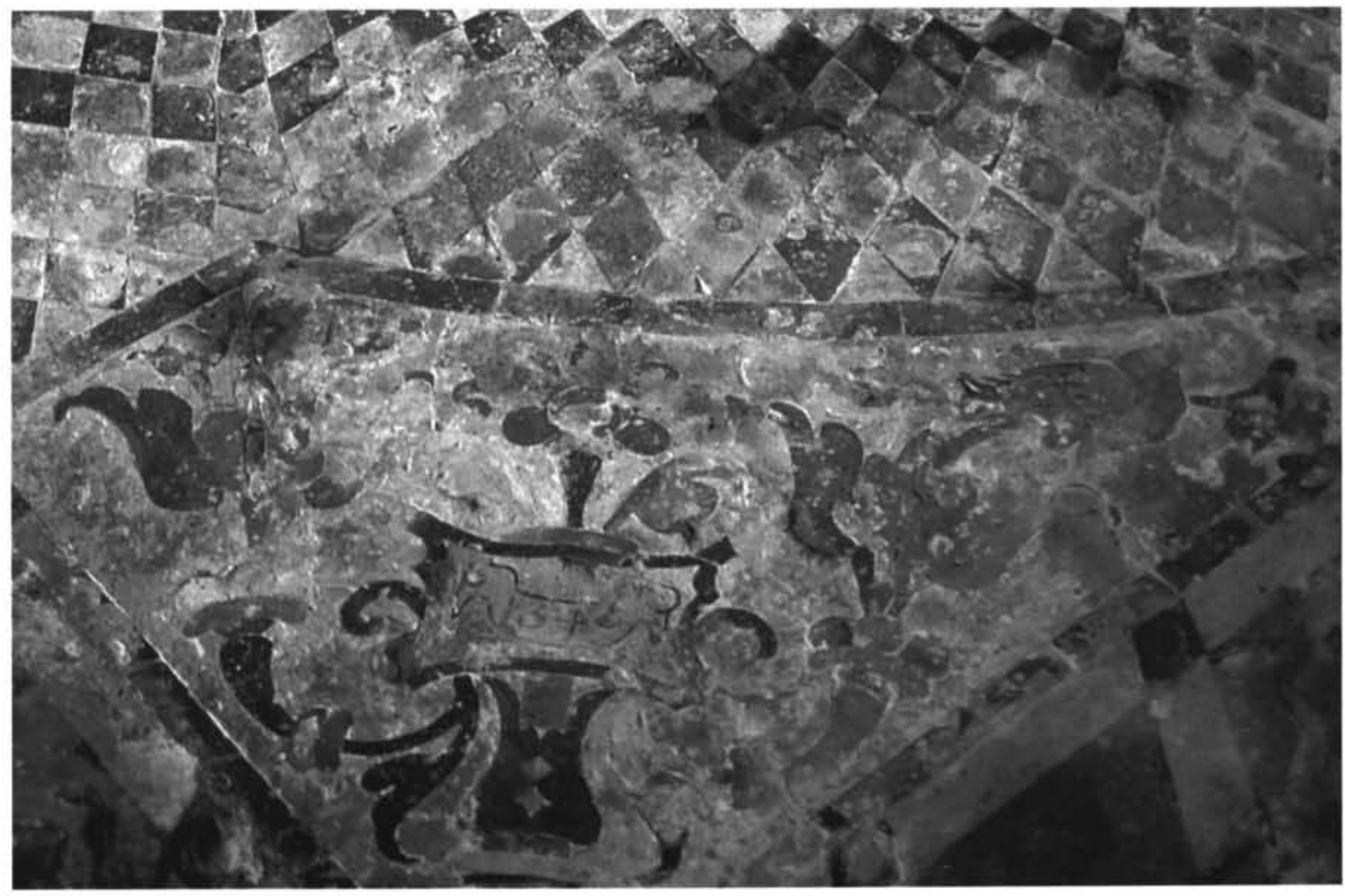

8. Detalle del mosaico del suelo del Pabellón de Carlos V.

Ángulo derecho próximo a la entrada, con la cartela que contiene la fecha de terminación del edificio, 1546. 


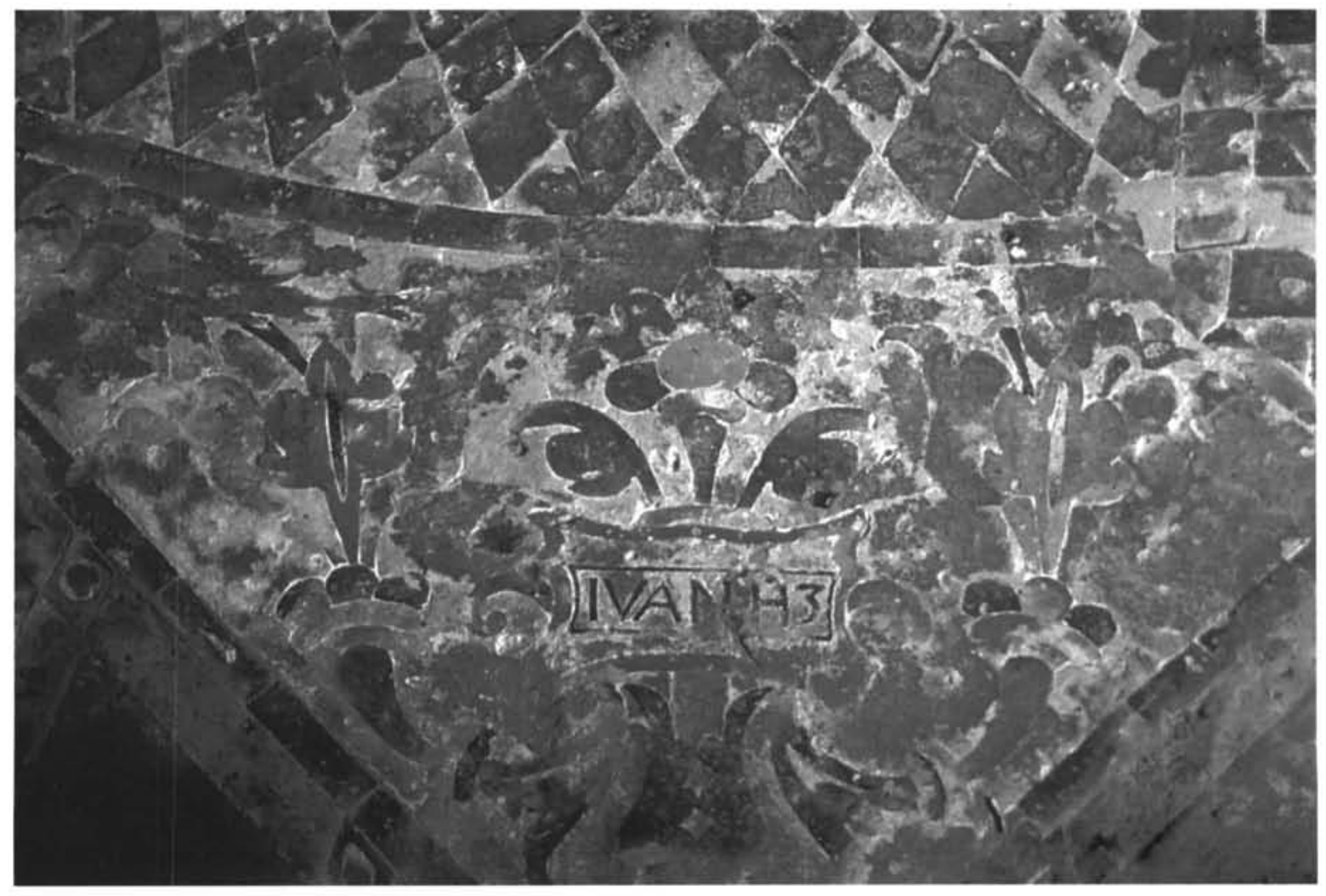




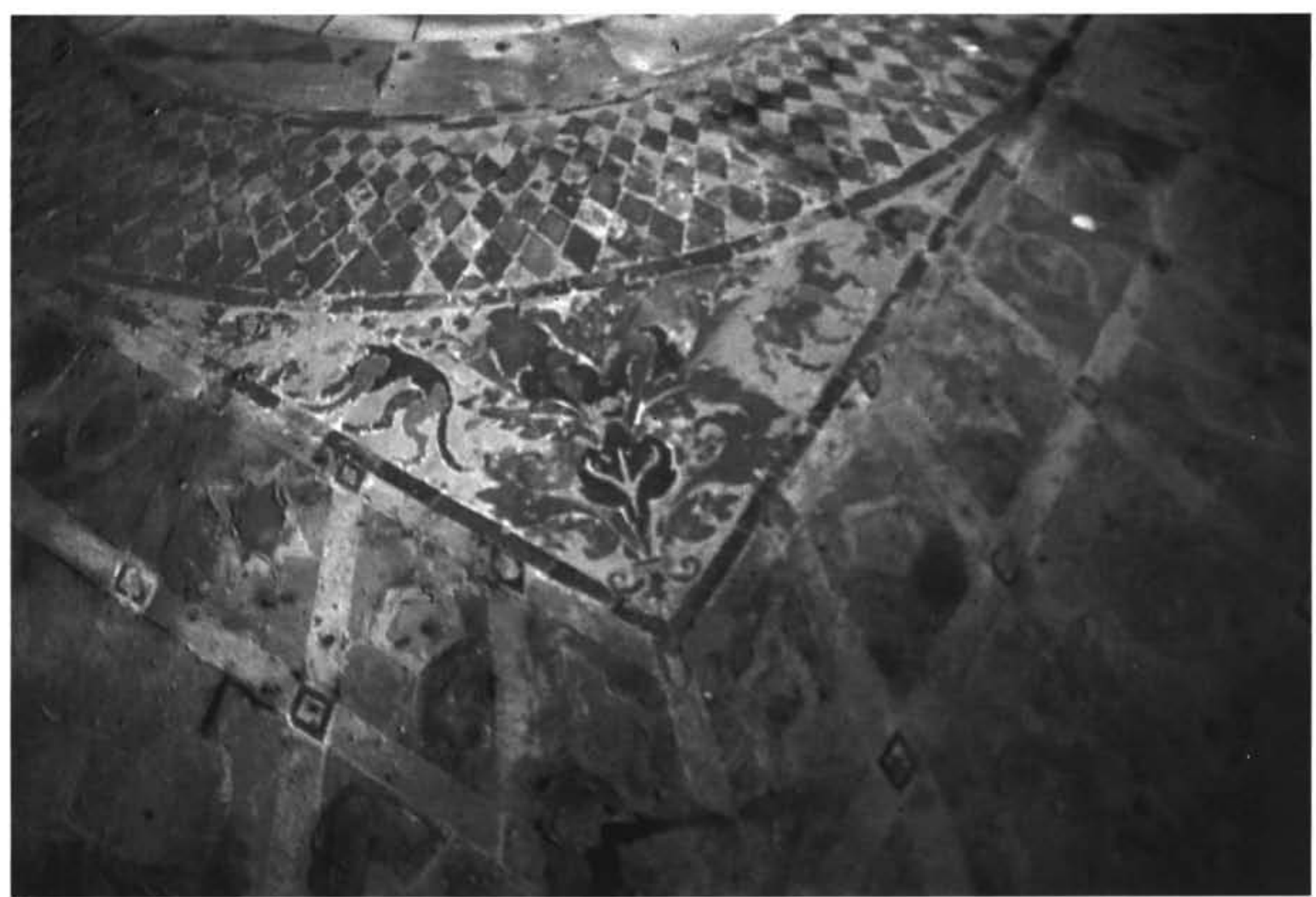

10. Ángulo derecho próximo al fondo, mostrando la composición completa del triángulo exterior al anillo de mosaico. 


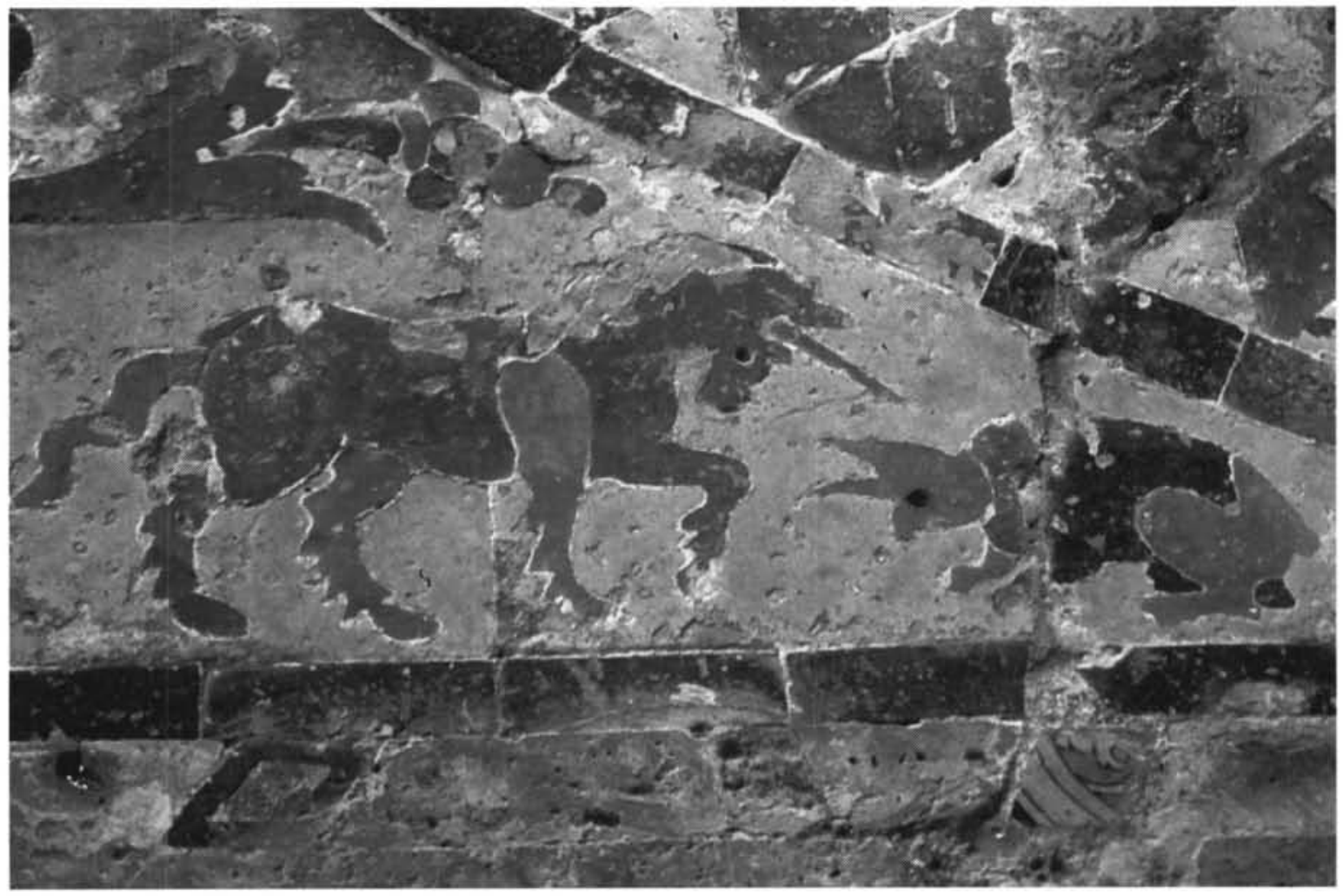




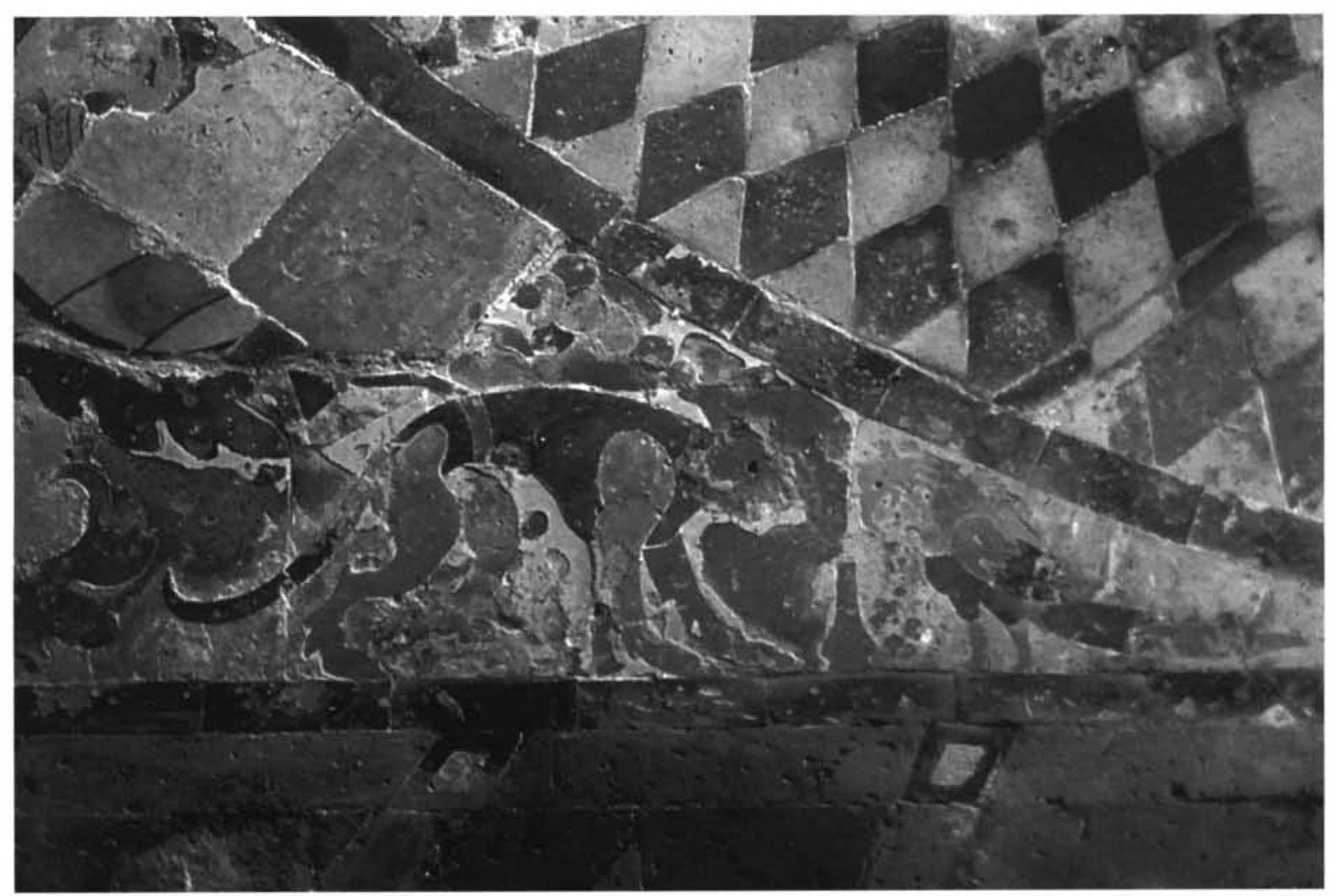

12. Ángulo izquierdo próximo al fondo, detalle. 


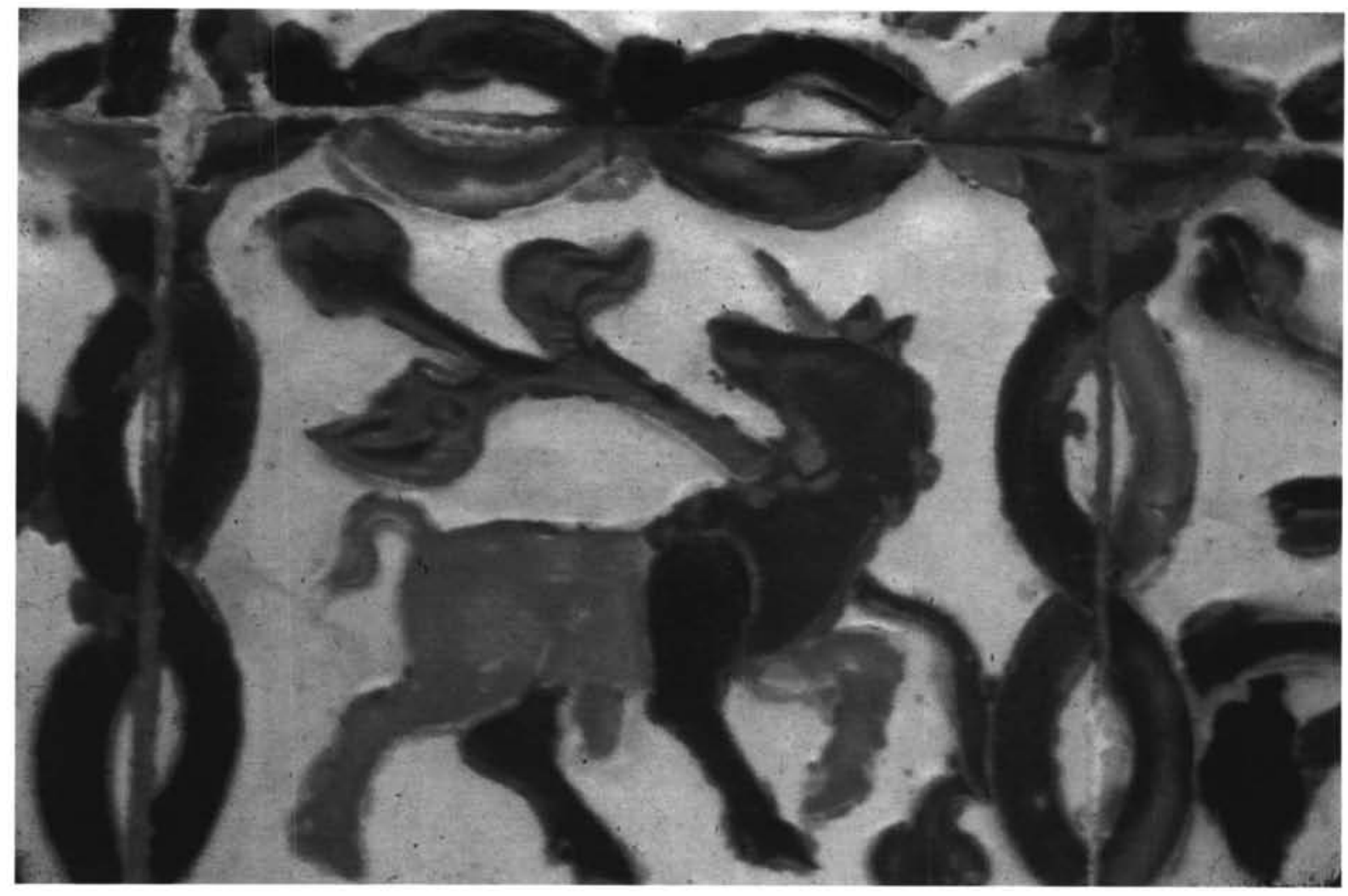




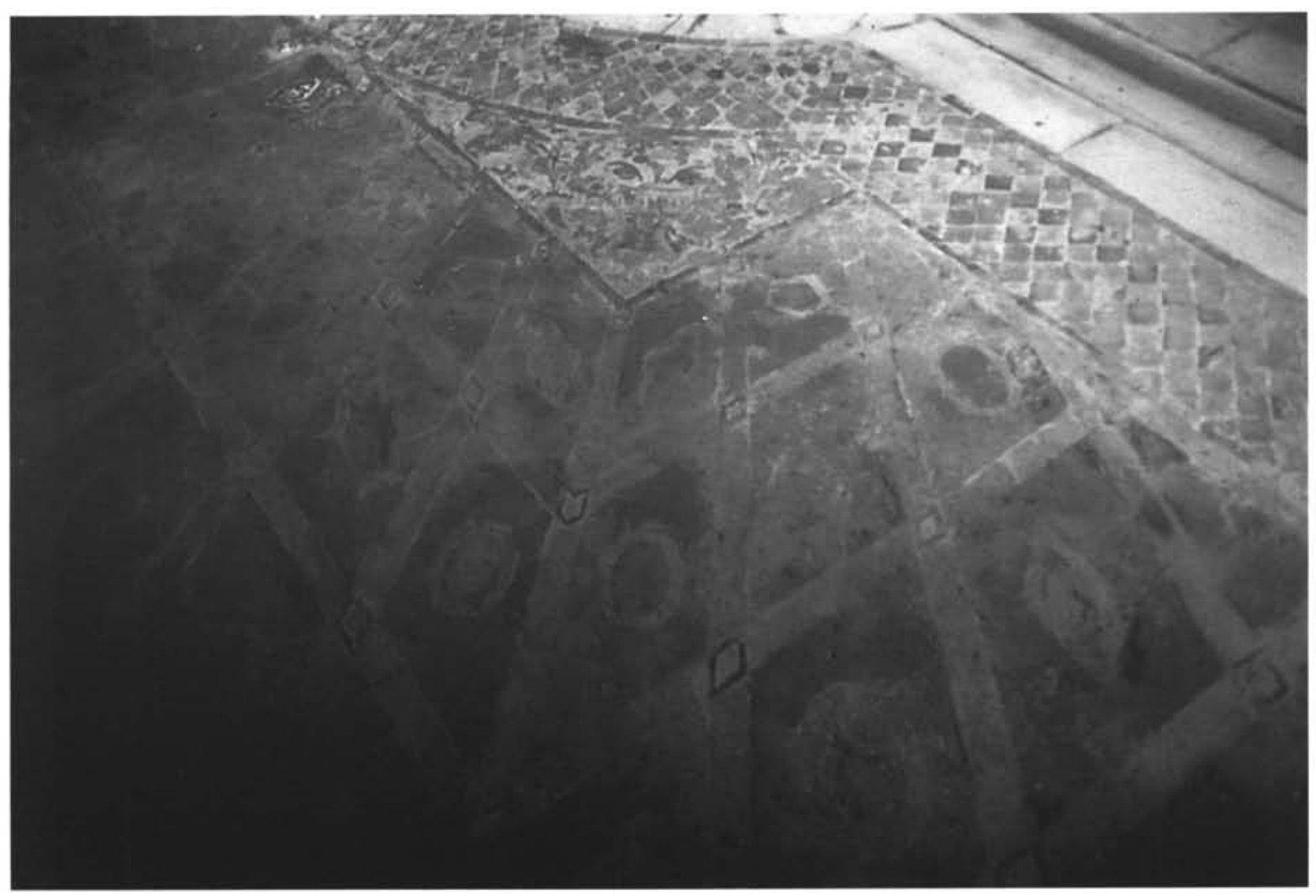

14. Suelo del Pabellón de Carlos V. Detalle del cuadrado exterior, con la olambrilla geométrica de barros de distinto color. 


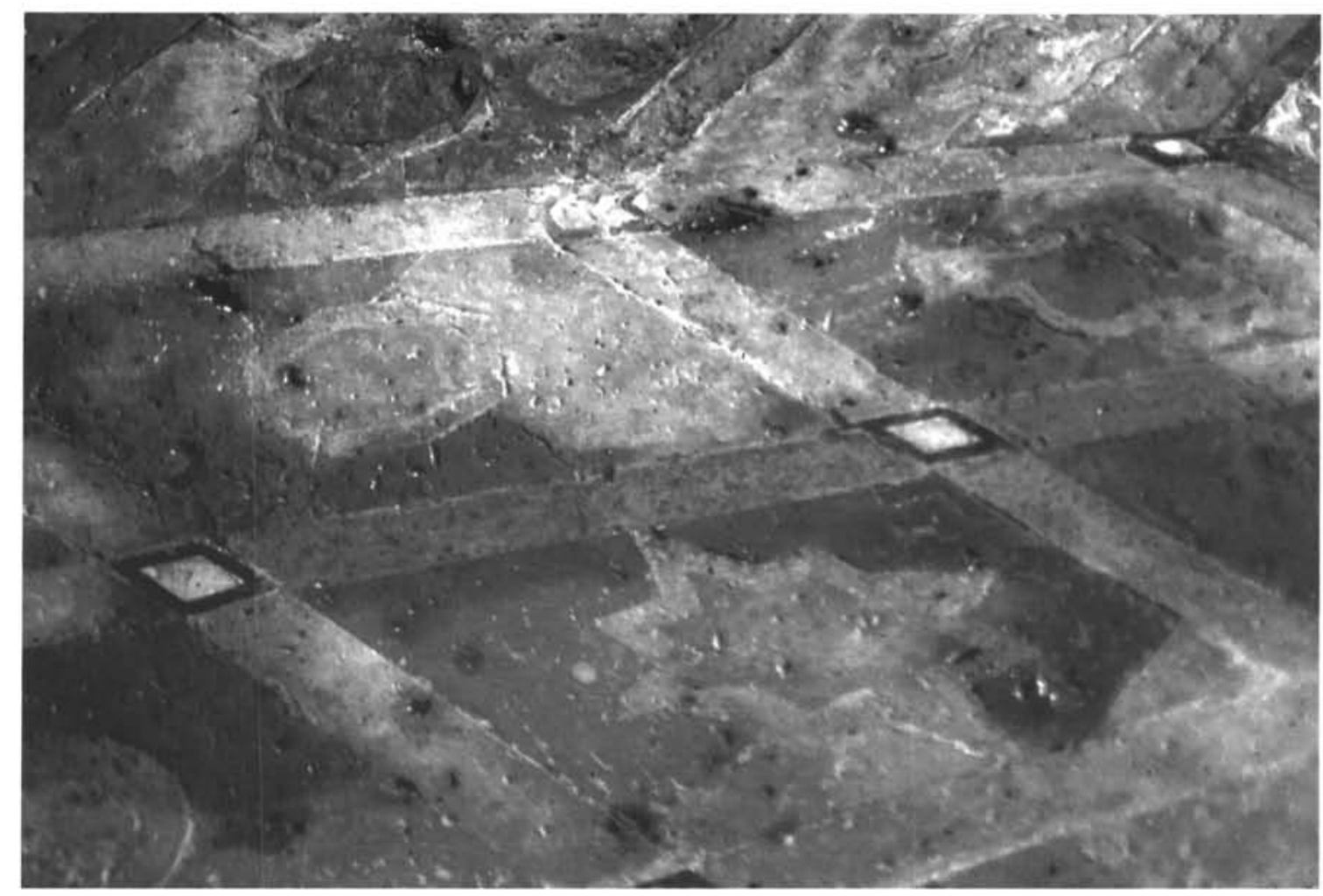

15. Detalle de la figura anterior, donde se aprecian las redes geométricas de barro sin esmaltar, con los pequeños azulejos esmaltados en los cruces de las líneas. 